\title{
Electron Diffraction Imaging of Molecular Nanostructures and Molecular Motions
}

\author{
J.M. Zuo, ${ }^{1}$ K. Ran ${ }^{1,2}$ \\ ${ }^{1}$ Department of Materials Science and Engineering and Materials Research Laboratory, University \\ of Illinois at Urbana-Champaign, 1304 W Green Street, Urbana, IL 61801 \\ ${ }^{2}$ Department of Electronics, Peking University, Beijing, P. R. China, 100871
}

Atomic resolution imaging of single molecules is a grand challenge in nanosciecne where direct observation at Angstrom resolution is the ultimate goal in nanostructure characterization. However, atomic resolution electron imaging is limited by the undesirable effects induced by electron beam irradiation. Thus, reducing the irradiation damage has been the driving force for the development of lower acceleration voltage, aberration corrected transmission electron microscopy (TEM) and scanning transmission electron microscopy. Here we will report progress made at University of Illinois on electron diffraction and imaging of molecular nanostructures using $\mathrm{C}_{60}$ molecules confined in single wall carbon nanotubes (peapods) as model system.[1]

We focused on the range of molecular motions of the vdW bonded $\mathrm{C}_{60}$ molecules that can be stimulated by the electron beam irradiation. We observed single fullerene's jump in a defective zigzag $\mathrm{C}_{60}$ molecular chain, back and forth translation of a cluster of $\mathrm{C}_{60} \mathrm{~S}$ in a partially filled $\mathrm{CNT}$, pickup of $\mathrm{C}_{60}$ molecules, and rotation of a zigzag chain of $\mathrm{C}_{60} \mathrm{~s}$ accompanied by deformation of the host CNT (Figure 1).

Using a simple model of vdW potential among $\mathrm{C}_{60}$ molecules and the host $\mathrm{CNT}$, we estimated the activation energies for some of the molecular motions, ranging from $\sim 0.3 \mathrm{eV}$ to $0.7 \mathrm{eV}$ (an example is shown in Figure 1). The lowest activation energy is associated with a single $\mathrm{C}_{60}$ detachment at the end of a $\mathrm{C}_{60}$ molecular chain, while the largest activation energy is associated with the molecular jump at a defect site. This demonstrates that molecular vacancies can lead to lower activation energy.

We calculated the probability of electron beam induced breakdown of $\mathrm{vdW}$ bonds of $\mathrm{C}_{60}$ molecules. We found that one-dimensional confinement can significantly increase the energy threshold for breaking the bonds. For imaging confined $\mathrm{C}_{60} \mathrm{~s}$, the optimum electron energy predicted is $\sim 60 \mathrm{kV}$.

Using above knowledge, we acquired electron diffraction patterns from $\mathrm{C}_{60}$ molecular chains using coherent nanoarea electron diffraction [2-3]. We demonstrated that diffraction peaks from $\mathrm{C}_{60}$ molecules can be recorded when the molecules are ordered and confined in small diameter CNTs. The diffraction patterns coupled with recorded electron images allow us to image the $\mathrm{C}_{60}$ molecules with significantly improved contrast and resolution [4-7].

\section{References}

[1] B. W. Smith et al., Nature 396 (1998) 323.

[2] M. Gao et al., Appl. Phys. Lett. 16 (2003) 2703. 
[3] J. M. Zuo et al., Microsc. Res. and Tech. 64 (2004) 347.

[4] J. M. Zuo et al., Science 300 (2003) 1419.

[5] W. J. Huang et al., Nat. Phys. 5 (2009) 129.

[6] W. J. Huang et al., Ultramicroscopy 107 (2007) 1159.

[7] This work was supported by NSF of China (60728102 and 60925003) and DOE (DEFG0201er45923). K. R. acknowledges fellowship support from CSC (China). Microscopy was carried out at the Frederick Seitz Materials Research Laboratory Central Facilities, University of Illinois.
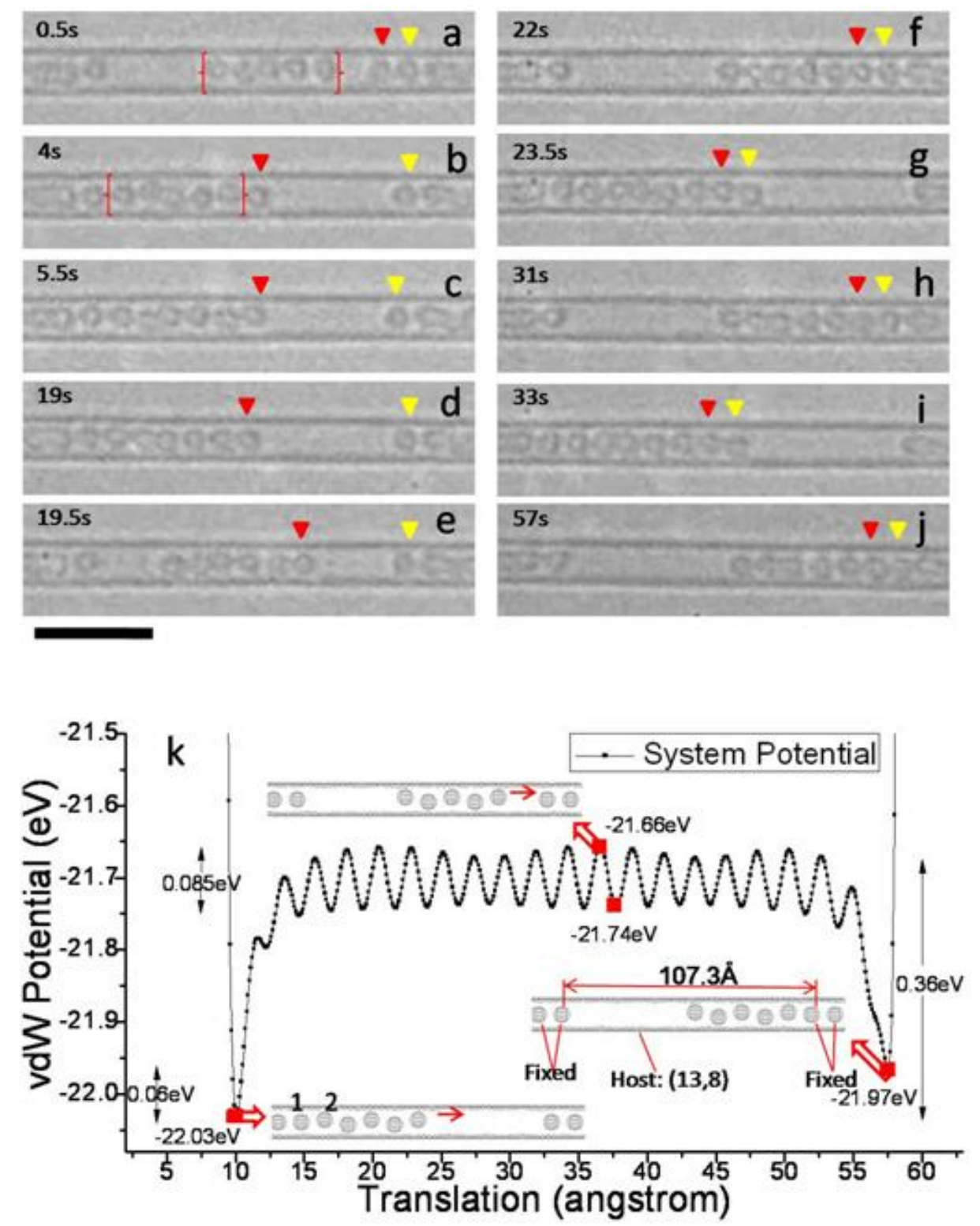

FIG. 1. (a-j) Time series of TEM images captured from a video showing back and forth translations of a small chain of $\mathrm{C}_{60} \mathrm{~s}$. The recording lasted for $1 \mathrm{~min}$, and 120 frames in total were recorded with an exposure time of $0.5 \mathrm{~s}$. The scale bar is $5 \mathrm{~nm}$. (k) Calculated van der Waals potential energy for the translation of a chain of $5 \mathrm{C}_{60} \mathrm{~s}$ inside a CNT. The structural models used for the calculation is shown in insets for three different positions. 